\title{
Influence of different anesthetics on some physiological parameters and stress markers during and after surgery of a dog
}

\author{
Dimiter Dimitrov, Konstantin Aminkov, Georgi \\ Marinov
}

University of Forestry, Faculty of Veterinary Medicine, Sofia, 1000, Bulgaria;

\begin{abstract}
Surgical operations on animals are stressful for their organism. This results in changes in several physiological parameters and stress markers. The application of different anesthetics and different anesthetic schemes has a different impact on the organism's response to stress response and changes of physiological parameters. The study of the effects of different anesthetics on animal organism will facilitate the prediction of the animal's responses to surgical procedures and increase patient's safety.
\end{abstract}

\section{Introduction}

Like humans, animals may react to stress by the stimulation of the sympatho-adrenal-medullary (SAM) axis and the hypothalamic-pituitary-adrenal (HPA) axis. Activation of these systems causes changes in physiological parameters such as heart and respiratory rate and cortisol, ACTH, and neuropeptide secretion. Although essential for coping with acute changes in the body's homeostasis, stress and particularly prolonged stress reactions during surgery can be detrimental (Sapolsky et al. 2000). Pain, as well as surgery, can induce a stress response (Desborough 2000, Giannoudis et al. 2006). In an attempt 
to identify painful processes in animals, various authors have proposed the evaluation of the serum cortisol and plasma catecholamine levels, thus identifying techniques and safe anesthesia for the wellbeing and ethics of animal handling (Tranquilli et al., 2007; Mattos Junior et al., 2009). They have found that the type of anesthesia may affect the intra-operative and postoperative stress response (Crozier 1994, Mehandzhiyski N. et al. 2007).

The stress reaction induced by surgery is generally proportional to the degree of tissue trauma (Marana et al. 2003, Chernow et al.1987, Horta et al. 2015). Postoperative stress and pain intensity can also be affected by other factors such as surgical skill and techniques, analgesic protocol, and complications (Michelsen et al. 2012, Devitt at al. 2005, Mastrocinque et al. 2012, Mastrocinque et al. 2003 ). Identifying and preventing prolonged pain and stress in surgically treated patients is therefore important for the animal welfare and optimal recuperation (Epstein et al. 2015, Mathews 2000). The assessment of the neuroendocrine response has been accepted as a reasonable surrogate for surgical stress and pain in animal models and it has been found that cortisol is a sensitive blood marker for the evaluation of pain (Dart, 1999; Ko et al., 2000; Yoder and Wolf, 2005). In dogs, a significant difference in the stress response in animals with and without surgery has been observed. In animals undergoing general anesthesia without surgery, a short-term increase in cortisol levels and ACTH plasma concentrations has been observed, whereas in animals with surgery there has been a significantly higher increase in the plasma levels of the same hormones (Benson et al., 2000).

\section{a2 agonists}

Different pre-medications are used in veterinary practice. Several studies show that $\alpha 2$ agonists like xylazin, clonidine and medetomidine have an influence on the stress response, in particular on cortisol levels (Benson 1991, Frank 1992, Scheinin 1989). Applied alone or in combination with other anesthetics affects changes in the neuroendocrine response. Medetomidine doesn't prevent the increase of stressrelated cortisol in dogs (Ambrisko et Hikasa 2003). Maze et al. (1997) reported that high-doses of I.M. Dexmedetomidine decreases basal Cortisol and ACHT 3 hours after their application. In conclusion, only high doses of Dexmedetomidine inhibit the adrenal steroid synthesis. Some studies show that premedication with Dexmedetomidine during ovariohysterectomy in dogs decreases the serum levels of Cortisol (Benson 1991, Ko 2000). Comparative studies in dogs during ovariohysterectomy with premedication with Medetomidine or Acepromazine should cause statistically significant decrease of the levels of Catecholamine and Cortisol in the Medetomidine group (Väisänen M et al. 2002). Another study focused on the influence of Benzodiazepines showed that oxazepam given a few hours before blood sampling, may suppress cortisol levels. It may be postulated that this effect is mediated through GABAergic receptors inhibiting the hypothalamic release of corticotropin releasing factor (CRF) Gram LF 1986.

\section{Dissociative agents}

Ketamine is a dissociative agent. It is used in combination with other premedication products to deliver short-term anesthesia in humans (White 1982) and animals (Moens 1990, Verstegenn1989). Ketamine also has analgesic effects, most probably related to its nature as the NMDA receptor antagonist and partial opioid receptor antagonist (Hustveit 1995). Application of Ketamine influences the endocrine 
response of the body (Adams 1997). Ambrisko (2005) showed that Ketmine applied in Beagle dogs without any surgical intervention leads to increase of plasma Cortisol levels. A study conducted by Haddaf et al. (2000) found statistically non-significant increase of the plasma Cortisol levels during and after laparoscopic surgery in dogs anaesthetized with Xylazine and Ketamine.

\section{Epidural anesthesia}

Changes in hormonal response during epidural anesthesia and surgical stress have been studied by numerous authors and results are contradictory (Kaufman 1982, Kaufman 1984). The common conclusion (Covino 1988) is that epidural anesthesia directly influences the endocrine response. (Moore 1994, Cousins 1984). Nociceptive impulses that run through the spinal cord activate the endocrine impulses to different organs. Surgical interventions lead to impulses that differ in strength and degree in different organs. Simeonova (2007) conducted a comparative study to evaluate changes in Cortisol levels in dogs during three anesthetic schemes (with halothane, balanced and epidural). Statistically significant increase in Cortisol on the 140 minute $(209.2 \mathrm{nmol} / \mathrm{l})$ was registered for the epidural anesthesia with Lidocaine. Other authors (Aminkov et. al., 2007) have confirmed the above results and reported significant increase in cortisol during surgery and epidural anesthesia with Xylazine and Ketamine. Oyama 1971 reported insignificant changes in Cortisol levels during epidural anesthesia. a2 agonists applied via epidural infusion mediate analgesia through activation of presynaptic $\alpha 2$ adrenoceptors in the spinal cord (Buerkle 1998), which is associated with antinociceptive effects (Takano et al. 1993, Eisenach et al. 1989). Mehandzhiysky et al. (2014) compared the effects on cortisol and ACTH levels of two types of anesthesia: epidural with a mixture of xylazine and lidocaine and multimodal. They found significantly higher increases in both hormones in the group with epidural anesthesia.

Application of opioids via epidural infusion results in deeper and longer analgesia in comparison to their I.M or I.V. application. Local anesthetics during epidural application affect both nociceptive receptors and nociceptive pathways; on the other hand, opioids selectively block only nociceptive impulses (Cousins 1979). For pain control, epidural application of opioids alone is the choice of preference, while during surgical interventions they are combined with local anesthetics via epidural application. (Popilskis 1993). Pain is a complex symptom including pathophysiological and psychological components and it is often challenging to recognize or interpret in animals (ACVA 1998). Research of patients undergoing surgery and anesthetized with different anesthesia regimens makes it possible to assess the potential of the study schemes to modulate the various aspects of the body's response to such a strong stimulus as surgical intervention. Due to the large number of opioid receptors within the spinal cord, epidural application of low doses of opioids results in deep analgesia (Yaksh et al.1976). Epidural application of opioids prolongs the anesthesia with minimal side effects (Quandt et al. 1996; Pascoe et al. 1993; Hendrix et al.1996). Intravenous application of Fentanyl suppresses breathing for a few hours and increases PaCO2 (Hug et al. 1979). Different authors report additive or synergic effects of combining opioids and local anesthetics in epidural anesthesia in humans (Solomon et al. 1994; Maurette et al. 1993), in dogs (Quandt et al. 1996; Hendrix et al. 1996; Torske et al.1999) and other animals (Akerman et al. 1988).

Epidural application of local anesthetics together with opioids or a2 agonists is used for surgical interventions in the area caudal of the belly button in dogs and provides analgesia during and after surgery. 
Application of $\alpha 2$ agonists similar to opioids may influence nociceptive processes by activation of the $\alpha 2$ receptors in the central and peripheral neurous system (Reddy 1980). Epidural application of $\alpha 2$ agonists mediates analgesia by activation of pre-synaptic $\alpha 2$ adrenoreceptors within the spinal cord (Buerkle 1998). The main side effects that can be caused by epidural application of $\alpha 2$ agonists are respiratory acidosis, bradycardia, hypotension and hypoxemia (Skarda et al 1990).

\section{Changes within the ECG parameters during anesthesia and surgical intervention}

Application of Atropine in animals significantly affects the heart rate and cardiac rhythm (Kantelip et al. 1985; Richards et al. 1989; Muir 1978). The standard I.M application of Atropine in doses of 0,02 - 0,04 $\mathrm{mg} / \mathrm{kg}$ results in a typical cardiac response: increased cardiac atrium contractions, increased sinus rhythm and accelerated conduction through AV node (Kantelip et al. 1985; Richards et al. 1989). Xylazine plus ketamine is an injectable anesthesia used in small animal surgeries. Xylazine is one of the alpha-2 adrenergic agonists and produces dose-dependent sedation, analgesia and muscle relaxation. However, alpha-2 agonists alter cardiovascular function profoundly by causing bradycardia, hypertension followed by hypotension, decreased myocardial contractility, and dysarhythmias (Dart, 1999; Hall et al., 2001; Tranquilli et al., 2007; Plumb, 2008). Ketamine effects on the cardiovascular system are due to indirect cardiovascular stimulation. It has a similar effect on other systems similar to the sympatico-mimetic effect medicated by the central nervous system (Ivancovitch et al. 1974), the direct vasodilation of the smooth muscles vessels (Ivancovitch et al.1970) and the positive inotropic effect on the myocardium. Dogs anesthetized with Ketamine show increase in mid-arterial pressure, pulse and cardiac debit while the peripheral vessel resistance remains unchanged (Haskins et al.1975; Traber et al. 1977; Nakajima et al. 1978). Joon-ki Kim et al. (2004) studied the influence of the combination Ketamine-Xylazin in doses of $10 \mathrm{mg} / \mathrm{kg}$ and $2 \mathrm{mg} / \mathrm{kg}$ respectively applied in different ways: I.M.; I.V. and intratesticular during dogs castration. Results showed statistically significant decrease of the heart rate on the 30th and 45th minute $(p<0.005)$. Cardiac arrhythmias were significantly reduced in the group with intratesticular application. Mehandzhiyski et al.2008 studied the effects of anesthesia with Atropine, Xylazin and Ketamine on heart electrophysiology during surgical intervention. The authors reported atrial extrasystoles, extension of conduction time from SA to $A V$ node, increased time for ventricular depolarization, reduction of the repolarization potential. Atalan et al. (2002) carried out a comparative study on ECG parameters in dogs with two anestethic schemes. The first group was treated with Xylazin and Ketamine and the second group - with Acepromazine, Butorphanol and Ketamine. The results showed sinus arrhythmia and bradycardia in the first group of dogs.

The effects of local anesthetics are a result of the direct electro-physiologic and mechanic effects on the myocardium and peripheral circulation and of the anesthetic effects on the autonomous nervous system. Greene et al. 1995 studied the influence of epidural application of 0,2mg/kg of Xylazin in dogs, initially anesthetized with isoflurane. The authors didn't record any statistically significant changes in the ECG parameters and concluded that Xylazin applied in dogs anesthetized with isoflurane had minimal cardiovascular risk. Simeonova (2004) studied lumbosacral epidural anesthesia with $2 \%$ Lidocaine in dose $0.3 \mathrm{ml} / \mathrm{kg}$. in dogs and didn't register statistically significant changes in ECG parameters. Mehandzhiyski et al.(2009) compared the effects on the ECG parameters of general and epidural anesthesia during surgical intervention in dogs. Their results showed bradycardia and prolonged Q-T and P-R intervals. 


\section{Conclusion}

From the literature review, we can conclude that surgical interventions increase stress- related hormones, ACHT and Cortisol levels in particular. Different authors have studied the influence of Xylazin-Ketamine anesthesia on hormone secretion without surgery and results have showed that Xylazin suppresses the release of stress hormones, while Ketamine does not prevent or decrease the secretion of the same hormones. Combined application of Xylazin and Ketamine in standard doses increases ACTH and Cortisol levels, more significantly in patients with surgery and less in patients without surgery. Epidural anesthesia reduces, to a different extent stress-related endocrine response of the body according to literature. Stress response is influenced by the strength of pain stimulation. Comparing the two anesthetic schemes it has been confirmed that the one with epidural anesthesia is always superior to the combined scheme XyzalinKetamine in terms of suppression of hormonal stress response.

The influence of the reviewed anesthetic schemes on the cardiac electro-physiological parameters can be summarized as follows; different authors have established that the use of an intravenous scheme containing Atropine, Xylazin and Ketamine leads to atrial extrasystoles, prolongation of conduction time from SA to AV node, prolongation of chamber depolarization time, decrease of repolarization potential; anesthetic schemes with epidural anesthesia lead to bradycardia and prolongation of some of the conductive times. The conclusion of these comparative studies is that epidural anesthesia is superior to general anesthesia with $\alpha 2$ agonists and dissociative agents in terms of cardiac electro-physiologic parameters.

Despite the fact that in most of the cases epidural anesthesia cannot be used alone it is the most desirable choice in terms of the cardiac patient's safety and reduction of the stress response of the body.

\section{References}

1. Aminkov B., Baichev Z., Mehandzhiyski N., Influence of epidural anesthesia on the neuroendocrine response of the bitch with ovariohysterectomy. Veterinary Medicine, Year XI, No 3-4, 2007: 46-48

2. Chernow B, Alexander HR, Smallridge RC, Thompson WR, Cook D, Beardsley D, Fink MP, Lake $\mathrm{CR}$, Fletcher JR. Hormonal responses to graded surgical stress. Arch Intern Med. 1987;147(7):1273-1278.

3. Crozier TA, Mueller JE, Quittkat D, et al. Total intravenous anaesthesia with methohexitonealfentanil or propofol-alfentanil: clinical aspects and hemodynamic, endocrine and metabolic effects. Anaesthesist 1994;43:594-604.

4. Dart C.M. Advantages and disadvantages of using alpha-2 agonists in veterinary practice. Aust. Vet. J. 1999;77:720-721.

5. Desborough JP. The stress response to trauma and surgery. Br J Anaesth. 2000;85(1):109-117.

6. Devitt CM, Cox RE, Hailey JJ. Duration, complications, stress, and pain of open ovariohysterectomy versus a simple method of laparoscopic-assisted ovariohysterectomy in dogs. J Am Vet Med Assoc. 2005;227(6):921-927. 
7. Epstein M, Rodan I, Griffenhagen G, Kadrlik J, Petty M, Robertson S, Simpson W. 2015 AAHA/AAFP pain management guidelines for dogs and cats. J Am Anim Hosp Assoc. 2015;51(2):67-84.

8. Giannoudis PV, Dinopoulos H, Chalidis B, Hall GM. Surgical stress response. Injury. 2006;37(Suppl 5):3-9.

9. Gram LF, Christensen P. Benzodiazepine suppression of cortisol secretion: a measure of anxiolytic activity?, Pharmacopsychiatry. 1986, 19(1):19-22

10. Hall L.W, Clarke K.W, Trim C.M. 10th ed. S. co. Iondon; 2001. Veterinary Anesthesia.

11. Horta RS, Figueiredo MS, Lavalle GE, Costa MP, Cunha RM, Araujo RB. Surgical stress and postoperative complications related to regional and radical mastectomy in dogs. Acta Vet Scand. 2015;57:34.

12. Ko J.C.H, Mandsager R.E, Lange D.N, Fox S.M. Cardiorespiratory responses and plasma cortisol concentrations in dogs treated with medetomidine before undergoing ovariohysterectomy. J. Am. Vet. Med. Assoc. 2000;217(4):509-514.

13. Marana E, Scambia G, Maussier ML, Parpaglioni R, Ferrandina G, Meo F, Sciarra M, Marana R. Neuroendocrine stress response in patients undergoing benign ovarian cyst surgery by laparoscopy, minilaparotomy, and laparotomy. J Am Assoc Gynecol Laparosc. 2003;10(2):159_ 165.

14. Mastrocinque S, Almeida TF, Tatarunas AC, Imagawa VH, Otsuki DA, Matera JM, Fantoni DT. Comparison of epidural and systemic tramadol for analgesia following ovariohysterectomy. J Am Anim Hosp Assoc. 2012;48(5):310-319.

15. Mastrocinque S, Fantoni DT. A comparison of preoperative tramadol and morphine for the control of early postoperative pain in canine ovariohysterectomy. Vet Anaesth Analg. 2003;30(4):220228.

16. Mathews KA. Pain assessment and general approach to management. Vet Clin Am Small Anim Pract. 2000;30(4):729-755.

17. Mehandzhiyski N., Baichev Z., Aminkov B. Effect of xylazine-ketamine anesthesia on hormonal distress during surgery. Experimental Pathology and Parasitology, 10/3, 2007; 10(3): 9-13

18. Mehandzhiyski N., Peev I., Aminkov B., Mehandzhiyska E. Electrocardiographic changes in venous and epidural anesthesia and ovariohysterectomy in female dogs. Collection of papers from the scientific conference "Traditions and Modernity in Veterinary Medicine" LTU 2009: 179185

19. Mehandzhiyski N., Peev I., Raichev I., Georgiev G.I., 2014. Influence of Epidural and Multimodal anesthesia on the dynamics of plasma concentrations of ACTH and Cortisol in female dogs with castration. Collection of papers from the scientific conference "Traditions and Contemporaryity in Veterinary Medicine" at LTU - Sofia, 110-117.

Mehandzhiyski N., Peev I., Raichev I., Mehandziyska E. Electrocardiographic changes in atropinxylazine-ketamine anesthesia and ovariohysterectomy in bitches. Collection of papers from the scientific conference "Traditions and Modernity in Veterinary Medicine" 2008, 143-147

20. Michelsen J, Heller J, Wills F, Noble GK. Effect of surgeon experience on postoperative plasma cortisol and C-reactive protein concentrations after ovariohysterectomy in the dog: a randomised trial. Aust Vet J. 2012;90(12):474-478.

21. Naddaf H.,Najafzade Varzi H., Sabiza S., FalahH. Effects of xylazine-ketamine anesthesia on plasma levels of cortisol and vital signs during laparotomy in dogs. Open Vet J. 2014; 4(2): 85-89.

22. Plumb D. 6th ed. Stockholm: Blackwell; 2008. Plumbs Veterinary Drug Hand Book; pp. 707-708. 807. 
23. Sapolsky RM, Romero LM, Munck AU. How do glucocorticoids influence stress responses? Integrating permissive, suppressive, stimulatory, and preparative actions. Endocr Rev. 2000;21(1):55-89.

24. Tranquilli W.J, Thurmon J.C, Grimm K.A. 4th ed. oxford: Blackwell; 2007. Lumb \& Jones' Veterinary Anesthesia and Analgesia.

25. Väisänen M., Raekallio M., Kuusela E., Huttunen P., Leppäluoto J., Kirves P., Vainio O. Evaluation of the perioperative stress response in dogs administered medetomidine or acepromazine as part of the preanesthetic medication. Am J Vet Res. 2002 Oct;63(10):1358.

26. Yoder B, Wolf J.S. Canine model of surgical stress response comparing standard laparoscopic, microlaparoscopic, and hand-assisted laparoscopic nephrectomy. Urology. 2005;65(3):600-603.

\section{Corresponding author:}

Dimiter Dimitrov, DVM, PhD

Faculty of Veterinary Medicine, University of Forestry,,

"Kliment Ohridsky" str.,10 1756

Sofia, Bulgaria

email: mitko_3d@hotmail.com 